\title{
Improved Gabor Filter for Extracting Texture Edge Features in Ultrasound Kidney Images
}

\author{
P.R. Tamilselvi \\ Computer Technology, Kongu Engineering College \\ Perundurai - 52, Erode, Tamilnadu, India \\ E-mail: selvipr2003@yahoo.com \\ P.Thangaraj \\ School of Computer Technology and Applications \\ Kongu Engineering College \\ Perundurai - 52, Erode, Tamilnadu, India \\ E-mail: deansca@kongu.ac.in
}

\begin{abstract}
The diagnosis of urinary tract calculi begins with a focused history of calculi, evolution of its symptoms and the duration. Computer assisted approaches for analyzing the images have increased since the manual interpretation of the image is a time consuming process and susceptible to human errors.

The proposed system develops a multi-scale wavelet based Bayesian speckle suppression method for ultrasound kidney images. The logarithmic transform of the original image is analyzed into the multi-scale wavelet domain. The subband decompositions of ultrasound images have significantly non-Gaussian statistics that are best described by families of heavy-tailed distributions. Bayesian estimators are designed to exploits these statistics.

Ultrasound (US) is increasingly considered as a viable alternative imaging modality in computer-assisted Kidney segmentation and disease diagnosis applications. Automatic Kidney segmentation from US images, however, remains a challenge due to speckle noise and various other artifacts inherent to US. This paper, design intensity invariant local image phase features, obtained using improved Gabor filter banks, for extracting edge texture features that occur at core and intermediate layer interfaces. The proposed model does the extension of phase symmetry features to modified gabor mode and their use in automatic extraction of kidney edge texture features from US normal and diseased patient images. The system functionality is proved qualitatively and quantitatively through experimentation for synthetic and real data sets. The localization feature value threshold is evaluated with the training samples of US images. The speckle noise error ratio with respect to the standard US image are compared and experimented.
\end{abstract}

Keywords: Kidney ultrasound images, Noise suppression, Edge Texture Analysis, Improved gabor filters

\section{Introduction}

Ultrasound (US) imaging is non-ionizing, fast, portable, inexpensive and capable of real time imaging, but unfortunately, US images typically contain significant speckle and other artifacts which complicate image interpretation and automatic processing was shown in Daanen, V., Tonetti, J., Troccaz, J(2004). If anatomical structures of interest could be visualized and localized with sufficient accuracy and clarity, US may in fact become a strong practical alternative imaging modality for selected applications in Kidney disease diagnosis, particularly for computer-assisted applications where the image can be processed to provide quantitative information on the kidney structures.

Imaging speckle is a phenomenon that occurs when a coherent source and a non-coherent detector are used to interrogate a medium, which is rough on the scale of the wavelength. Speckle occurs especially in images of the liver and kidney whose underlying structures are too small to be resolved by large wavelength ultrasound.

In a recent work, alpha-stable distributions, a family of heavy-tailed densities, are sufficiently flexible and rich to appropriately model wavelet coefficients of images in coding applications. In this paper, present a novel speckle suppression method for medical ultrasound images. The proposed processor consists of two major modules:

a) A sub band representation function that utilizes the wavelet transform and 
b) A Bayesian denoising algorithm based on an alpha-stable prior for the signal.

The texture is very important cue in region based segmentation of images. Texture features play a very important role in computer vision and pattern recognition. Texture Applications include industrial inspection, estimation of object range and orientation, shape analysis, satellite imaging, and medical diagnosis. In this paper, we study different definitions of texture that applied to US kidney images. The time- frequency transformed based method of texture discrimination, which is in turn based on Gabor filters is done. In Gabor transform, a signal can be represented in terms of sinusoids that are modulated by translated Gaussian windows. The resulting timefrequency decomposition is a suite of local Fourier transforms which displays any non stationary spectral trends. Here the interrelationship between individual transforms is well-understood and studied.

Texture can be seen in many images from multi-spectral remote sensed data to microscopic photography. Despite its importance, there is no unique and precise definition of texture. Each texture analysis method characterizes image texture in terms of the features it extracts from the image. Therefore, it depends not only studying the images but also on the goal for which the mage texture is used and the features that are extracted from the image. Something composed of closely interwoven elements or an organization of constituent particles of a body or substance; and the visual or tactile surface characteristics and appearance of something (e.g. fabric). An image texture is described by the number and types of its (tonal) primitives and the spatial organization or layout of its (tonal) primitives.

The basic pattern and repetition frequency of a texture sample could be perceptually invisible, although quantitatively present. In the deterministic formulation texture is considered as a basic local pattern that is periodically or quasi-periodically repeated over some area. An image texture may be defined as a local arrangement of image irradiances projected from a surface patch of perceptually homogeneous irradiances. Texture is characterized not only by the grey value at a given pixel, but also by the grey value 'pattern' in a neighborhood surrounding the pixel. The unit of texture is texels, and the repetitiveness of the texels determines the type of the texture and decides the texture analysis approach. The textures can be classified in various ways. One of the methods is to classify texture as perturbed or un perturbed, An "unperturbed texture" is basically an image formed of the exact periodic repetitions of some basic pattern in some definite orientation. Typical examples of the unperturbed textures are bricks, fish scales, stripes etc. On the other and, the "perturbed textures" are formed of texels arranged with arbitrary orientations and random overlaps. Most of the natural textures are the examples of perturbed textures.

\section{Related Works}

Speckle noise affects all coherent imaging systems including laser, SAR imagery, and ultrasound. Speckle may appear distinct in different imaging systems but it is always manifested in a granular pattern due to image formation under coherent waves.

The basic properties of speckle are described by J.W. Goodman(1976) while the main differences between ultrasound and laser speckle are discussed in J. G. Abbott and F. L. Thurstone(1979). A general model for speckle noise proposed by A. K. Jain(1989) was also used by Zong, A. F. Laine, and E. A. Geiser(1999). The proposed model formulates the ultrasound speckle removal problem starting with a speckle model. Generally, the effect of the additive component of the speckle in ultrasound images is less significant than the effect of the multiplicative component.

Manual identification of kidney surfaces in 2D US is time consuming and operator dependent, and thus limits clinical practicality. While some studies have shown some promise in automatically identifying the kidney surface based on intensity and gradient information (or a combination of both) these techniques were limited to 2D US, and remained sensitive to typical image variability and choice of processing parameters Mulet-Parada, M., Noble, J.A(2000). Barratt, D.C., Penney, P.G., Chan, S.K., Slomczykowski, M., Carter, T.J., Edwards, P.J., Hawkes, D.J(2006)proposed a method where prior knowledge of object structure appearance was incorporated. However, diseased surfaces do not have a continuous smooth surface and prior knowledge of fragment shape is mostly unavailable. Other approaches combined intensity and gradient-based techniques with multimodal registration of US to preoperative CT Dosil, R., Pardo, X.M., Fernandez-Vidal, X.R. (2006). However, preoperative CT requires additional time and expense and is not always considered necessary for diagnosis or treatment, so it is only available in selected cases.

The proposed system extends local phase based processing to US volumes using improved Gabor filters. Specifically, construct a local phase symmetry measure which produces strong responses at kidney surfaces and suppresses responses elsewhere. Then quantitatively investigate the accuracy of our technique in localizing kidney surfaces and assess the technique's ability to resolve edge texture of the kidney inner layer as well. The 
system analysis moves in the direction of display the kidney surfaces and its internal layers accurately localized using local phase features computed directly from 2D ultrasound image volumes.

\section{Noise Suppression using Bayesian estimator and Edge Texture extraction using Gabor bank filters}

\section{A. Noise Suppression using Bayesian estimator}

The system models design a formal Bayesian estimator that recovers the signal component of the wavelet coefficients in ultrasound images by using an alpha-stable signal prior distribution. The proposed processor is motivated by the modeling based on solid statistical theory, and it does not depend on the use of ad hoc thresholding and stretching parameters.

In a Bayesian framework, consider samples of random variables. The signal component is modeled according to a distribution with zero location parameter, while the noise component is modeled as a zero-mean Gaussian random variable. Our goal is to find the Bayes risk estimator that minimizes the conditional risk, which is the loss averaged over the conditional distribution for the given the set of wavelet coefficients.

The Bayes risk estimator under a quadratic cost function minimizes the mean-square error(mse) and is given by the conditional mean. The mse metric is defined for random variables that possess finite second-order moments. In this work, the signal component of the wavelet coefficients is modeled as an alpha stable random variable that does not have finite second-order statistics. Hence, use the absolute error as the loss function.

\section{B. Improved Gabor filters}

The purpose of edge detection is to capture the major axis of symmetry of a feature at some specified spatial scale.

Local phase information of a signal can be obtained by convolving the signal with a pair of band-pass quadrature filters (an odd filter and an even filter). Using two filters in quadrature enables the calculation of signal amplitude and phase at a particular scale (spatial frequency) at a given spatial location. The selection quadrature filters is the improved Gabor filter which can be constructed with arbitrary bandwidth. In order to obtain simultaneous localization of spatial and frequency information, analysis of the signal must be done over a narrow range (scale) of frequencies at different locations in the signal. This can be achieved by constructing a filter bank using a set of quadrature filters created from rescaling of the improved Gabor filter. Each scaling is designed to pick out particular frequencies of the signal being analyzed.

Symmetry information is investigated by looking at the points where the response of the even filter dominates the response of the odd filter taking the difference of their absolute values. The transfer function $(G)$ of an improved Gabor filter in the frequency domain is constructed as the product of two components: a one dimensional Log Gabor function that controls the frequencies to which the filter responds and a rotational symmetric angular Gaussian function that controls the orientation selectivity of the filter.

$$
G(\omega, \phi, \theta)=\exp \left(\frac{\left(\log \left(\omega / \omega_{0}\right)\right)^{2}}{2\left(\log \left(\kappa / \omega_{0}\right)\right)}\right) \times \exp \left(-\frac{\alpha(\phi, \theta)^{2}}{2 \sigma_{\alpha}^{2}}\right)
$$

\section{Texture edge detection of Ultrasonic Kidney Images}

The stages of texture edge detection are described below. The outputs of intermediate stages of processing are shown for each step for the US Kidney images

\section{Step 1 (Filtering)}

The Gabor filter and wavelet filter are applied along the set of all parallel lines of an image $\mathbf{I}$ in one direction (along all the vertical lines of the image). For a Gabor filter, the output is given as

$$
H_{k}\left(x_{c}, y\right)=I\left(x_{c}, y\right) * g_{k}
$$

where * indicates the convolution operator, $g_{k}$ represents Gabor filter with the parameter set $\mathrm{k}=(\mathrm{u}, \mathrm{l}), \mathrm{I}(\mathrm{xc}, \mathrm{y})$ represents the xc th column of the image $\boldsymbol{I}$, and $\boldsymbol{H}_{k}$ denotes $\mathrm{k}^{\text {th }}$ filter response. For a set of four Gabor filters the corresponding filtered images obtained are $\boldsymbol{H} 1, \ldots, \boldsymbol{H} 4$. Similarly, image $\boldsymbol{I}$ is filtered using two most commonly used DWT along the same directions. Let $\boldsymbol{H} 5, \mathbf{H 6}, \boldsymbol{H} 7$ and $\boldsymbol{H} 8$ denote the filter responses. Where $\boldsymbol{H} 5$ and $\boldsymbol{H} 6$ denote detail sub band of level-1 and level-2 decomposition of filter respectively and $\boldsymbol{H} 7$ and $\boldsymbol{H} 8$ denote detailed sub band of level-1 and level-2 decomposition of gabor filter respectively.

\section{Step 2 (smoothing)}

Filtered images obtained in step 1 are smoothed with asymmetric Gaussian filter. 
$\operatorname{Vi}(\mathrm{x}, \mathrm{y})=\operatorname{Hi}(\mathrm{x}, \mathrm{y}) * . \mathrm{L}(\mathrm{x}, \mathrm{y})$

where, $\mathrm{L}(\mathrm{x}, \mathrm{y})$ denotes a Gaussian filter with $\sigma_{x}=8 x \sigma_{y}$ if images are filtered along parallel vertical lines and $\sigma_{y}=8 x \sigma_{x}$, if images are filtered along parallel horizontal lines $\mathbf{V i}(\mathrm{x}, \mathrm{y})$ denotes the convolved output of $\mathrm{i}^{\text {th }}$ image where $\mathrm{i}=1, \ldots, 8$.

\section{Step 3}

Steps 1 and 2 are repeated to obtain filtered images in orthogonal direction. Let Fi(x, y) denote all the filtered images, where $\mathrm{i}=1, \ldots, 16$. Thus a 16 -dimensional vector $\mathrm{F}(\mathrm{x}, \mathrm{y})$ is obtained as:

$\mathbf{F}(\mathrm{x}, \mathrm{y})=[\mathrm{F} 1(\mathrm{x}, \mathrm{y}), \ldots, \mathrm{F} 16(\mathrm{x}, \mathrm{y})]$

\section{Step 4}

A one dimensional feature map $\Gamma$ over the vectors $\{\mathbf{F}(\mathrm{x}, \mathrm{y})\}$ is generated using the texture edge detection. For each pixel $(\mathrm{x}, \mathrm{y})$, the scalar index $\mathrm{M}(\mathrm{x}, \mathrm{y})$ of the reference vector closest to $\{\mathbf{F}(\mathrm{x}, \mathrm{y})\}$ is assigned

$$
M(x, y)=\arg \min _{i}\left\|F(x, y)-w_{i}\right\| \quad \text { for } w_{i} \in \Gamma
$$

In this way we transform the vector image to a scalar image. Feature map $\mathbf{M}$ is smoothed with a symmetric Gaussian

$\mathrm{E}(\mathrm{x}, \mathrm{y})=\mathrm{M}(\mathrm{x}, \mathrm{y}) * \mathrm{~L}(\mathrm{x}, \mathrm{y})$

Where, L(x, y) denotes Gaussian filter and $\mathbf{E}$ denotes a smoothed image. The feature map (scalar image) obtained from the filtered images of the image are depicted.

\section{Step 5 (canny edge detection)}

Canny's edge-detection method is applied to the smoothed feature map image E obtained in previous step to obtain the edge map.

\section{Step 6 (edge linking)}

The discontinuities in the edge map obtained using canny edge detection are linked using the similar algorithm. The steps of the algorithm are as follows:

(i) Find all the connected segments $\mathrm{c} 1, \mathrm{c} 2, \ldots, \mathrm{cb}, \ldots, \mathrm{cB}$ in the edge map obtained using canny edge detection. Where $\mathrm{cb}$ denotes a connected edge segment and $\mathrm{cB}$ denotes the total number of connected segments. Repeat step (ii) for $b=1, \ldots, B$

(ii) Find the end points of the connected segment $\mathrm{cb}$. Where, end points are the points, which contains only one edge pixel among their 8-Neighbors. Connect the end points of the cb th segment to the nearest edge pixel (other than pixels in $\mathrm{cb}$ th segment) or the boundary pixels of an image

(iii) Final edge map is obtained.

\section{Experimental Evaluation}

The experimentation of the ultrasound kidney images is conducted to quantitatively evaluate the performance of the proposed local phase and edge texture extraction for future kidney segmentation. The proposed algorithm was implemented in MATLAB. In addition to the quantitative speckle noise removal, the proposed model also present qualitative results for the texture extraction of the kidney image edges. The localization accuracy of kidney surface detection technique and assessing the accuracy of measuring relative inner layers of separation is a clinically relevant task for which the system uses 2D US imaging.

The Bayes risk estimator under a quadratic cost function minimizes the mean-square error (MSE). To quantify the achieved performance improvement the standard signal to noise ratio (SNR) is not adequate due to the multiplicative nature of speckle noise. Instead, a common way to achieve this in coherent imaging is to calculate the signal-to-MSE (S/MSE) ratio.

The results are summarized in Table 1. The measure corresponds to the classical SNR for the case of additive noise. From the table it can be easily seen that our proposed Bayesian approach exhibits the best speckle mitigation performance. Chart1 shows the comparison of various filters.

The table 2 shows the results of the edge detection of US Kidney images taken from different patients by applying improved gabor filters. The average pixel distance of the edge texture images gives identification for segmenting the US Kidney image proportions. The percentage of edges found using the improved gabor filters of 
the texture segmented portions of the US Kidney images are shown in the table 2 as well. Comparison is shown in chart 2 .

\section{Performance Results and Discussions}

For the implementation of the supervised texture segmentation algorithm, textured images from the Kidney dataset are used. Every textured image is preprocessed by Bayesian filter and averaging filters to minimize the effect of noise. (Shown Fig 1) If content of noise increases to the extent that image turns to be perturbed, then the Gabor filter scheme may not give optimum results. It has to be noted that Gabor filters are very sensitive to scale, orientation and frequency of the texture. If any of these are affected due to noise, then Gabor filter bank may not even detect the particular texture.

The filter bank parameter tuning, texture feature extraction and texture classifier design are performed as discussed in the previous sections. The multi-textured US kidney image is processed by the tuned filter bank and the textural features are extracted for each pixel position. The pixels are labeled to be belonging to certain texture classes as classified for further segmentation using seeded region growing method. The classified image is further processed where the pixel label is set to that which occurs maximum times in its $3 \times 3$ neighborhood. This ensures the removal of classification noise thereby enhancing the segmented image. Fig 2 shows results of US kidney image achieved by Gabor filter bank on natural textures.

\section{Conclusion}

The system model proposed, multi-scale nonlinear homomorphic method, effective does speckle suppression in ultrasound kidney images. The system deploys multi-resolution techniques to decompose kidney image into several scales by employing the 2-D wavelet transform. With this speckle is transformed from multiplicative into additive and its characteristics are differentiated from the signal characteristics in each decomposition level.

The proposed approach for accurate and fully automatic extraction of kidney surfaces directly in ultrasound volumes based on local phase symmetry image features that employ improved filters. Kidney surface localization accuracy assessed using normal kidney image standards and showed a maximum mean error of 0.12 $\mathrm{mm}$ and a low standard deviation across the sampled points of only $0.05 \mathrm{~mm}$. These errors were relatively independent of the depth of the edges of the kidney internal segment interface and of the inclination of the probe relative to the outer kidney surface.

The phase method has high localization accuracy even when the US beam is not perfectly normal to the kidney surface. The obtained results are encouraging for using local phase processed images in kidney assessment since the average accuracy required for such application is typically in the range of $1-2 \mathrm{~mm}$. The true analysis produced a noticeably smoother image of the kidney surface than previously reported 2D analysis. The image processing will be of special importance during the assessment of kidney disease diagnosis where good accuracy is needed to avoid elimination or redundant kidney inner layers. Furthermore, since there is no need to align the imaging plane with the anatomical area of interest, evaluation of the surface area can likely be performed more rapidly.

\section{References}

A. K. Jain. (1989). Fundamental of Digital Image Processing. Englewood Cliffs, NJ: Prentice-Hall.

Amin, D.V., Kanade, T., Digioia, A.M., Jaramaz, B. (2003). Ultrasound registration of the bone surface for surgical navigation. Journal of Computer Aided Surgery 8, 1-16.

Barratt, D.C., Penney, P.G., Chan, S.K., Slomczykowski, M., Carter, T.J., Edwards, P.J., Hawkes, D.J. (2006). Self calibrating 3D-ultrasound-based bone registration for minimally invasive orthopaedic surgery. IEEE Transactions on Medical Imaging 25, 312-323.

Chia-Hsiang Wu, Yung-Nien Sun. (2006). Segmentation of kidney for ultrasound B-mode images with texture-based classification, Computer Methods and programs in biomedicine.

Daanen, V., Tonetti, J., Troccaz, J. (2004). A fully automated method for the delineation of osseous interface in ultrasound images. In: Barillot, C., Haynor, D.R., Hellier, P. (eds.) MICCAI 2004. LNCS, vol. 3216, pp. 549-557. Springer, Heidelberg.

Dosil, R., Pardo, X.M., Fernandez-Vidal, X.R. (2006). Data driven synthesis of composite feature detectors for 3D image analysis. Journal of Image and Vision Computing, B225-238.

J. G. Abbott and F. L. Thurstone. (1979). “Acoustic speckle: Theory and experimental analysis," Ultrason. Imag., vol. 1, pp. 303-324 
J.W. Goodman. (1976). "Some fundamental properties of speckle,” J. Opt. Soc. Amer., vol. 66, pp. 1145-1150, Nov..

June Xie, Yifeng Jiang, and Hung-tat Tsui. (2005). Segmentation of Kidney from Ultrasound Images Based on Texture and shape priors, IEEE Trans, Med, Imag. Vol.34, No.1, January.

June Zhang, Jiulun Fan. (2006). Medical Image Segmentation based on Wavelet transformation and watershed Algorithm, IEEE Int. Conference on information acquisition, Aug.

K. Bommanna Raja, M.Madheswaran and K.Thyagarajah. (2007). "Analysis of Ultrasound kidney Images using Content Descriptive Multiple Features for Disorder Identification and ANN based classification", Proceedings of the International Conference on Computing; Theory and applications., IEEE computer Society.

Mohamed Roushdy. (2006). Comparative study of Edge detection Algorithms Applying on the Grayscale Noisy Image Using Morphological Filter, GVIP Journal, vol. 6, Issue 4, December.

Mulet-Parada, M., Noble, J.A. (2000). 2D+T boundary detection in echocardiography. Medical Image Analysis 4(1), 21-30.

S. G. Mallat. (1989). "A theory for multiresolution signal decomposition: The wavelet representation," IEEE Trans. Pattern Anal. Machine Intell., vol.11, pp. 674-692, July.

S.Poonguzhali and G.Ravindran. (2008). Automatic classification of Focal Lesions in Ultrasound liver images using combined texture features, Information Technology Journal 7 (1), 205-209.

X. Zong, A. F. Laine, and E. A. Geiser. (1999). "Speckle reduction and contrast enhancement of echocardiograms via multiscale nonlinear processing," IEEE Trans. Med. Imag., vol. 17, pp. 532-540, Aug. 1998. Washington, DC, June.

Table 1. Speckle Noise Suppression with multiple Filters in US Kidney Images

\begin{tabular}{|c|c|c|c|}
\hline Filters/ Error & $\begin{array}{l}\text { Bayesian } \\
\text { Filter }\end{array}$ & $\begin{array}{l}\text { Wiener } \\
\text { Filter }\end{array}$ & $\begin{array}{l}\text { Threshold } \\
\text { Filter }\end{array}$ \\
\hline Signal to noise error & 16.21 & 13.23 & 14.56 \\
\hline Signal to Mean Square Error & 21.32 & 18.76 & 18.88 \\
\hline
\end{tabular}

Table 2. Results of the Edge detection in US

\begin{tabular}{|c|c|c|c|}
\hline Patients & $\begin{array}{c}\text { Training } \\
\text { Sample1 } \\
(22)\end{array}$ & $\begin{array}{c}\text { Training } \\
\text { Sample 2 }\end{array}$ & Training \\
& $(17)$ & 10.23 \\
\hline Average distance [pix] & 9.36 & 11.06 & 84.46 \\
\hline Edges found using & 78.25 & 83.50 & \\
\hline
\end{tabular}




\section{Figures}

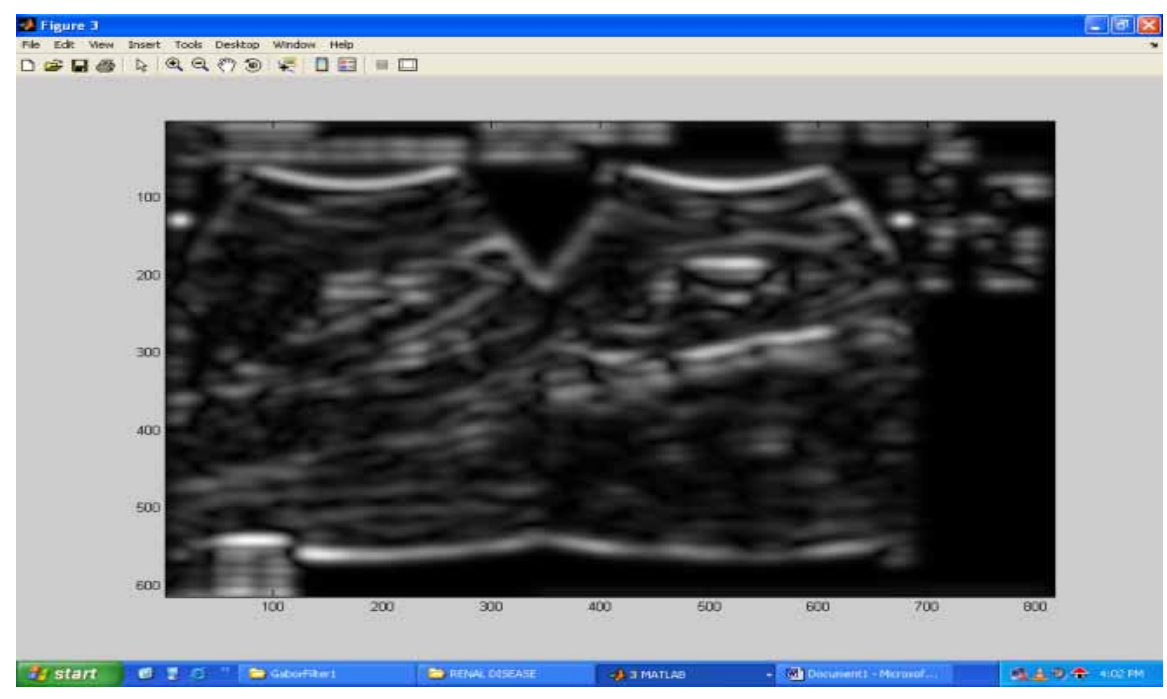

Figure 1. US Kidney Image with noise removal feature

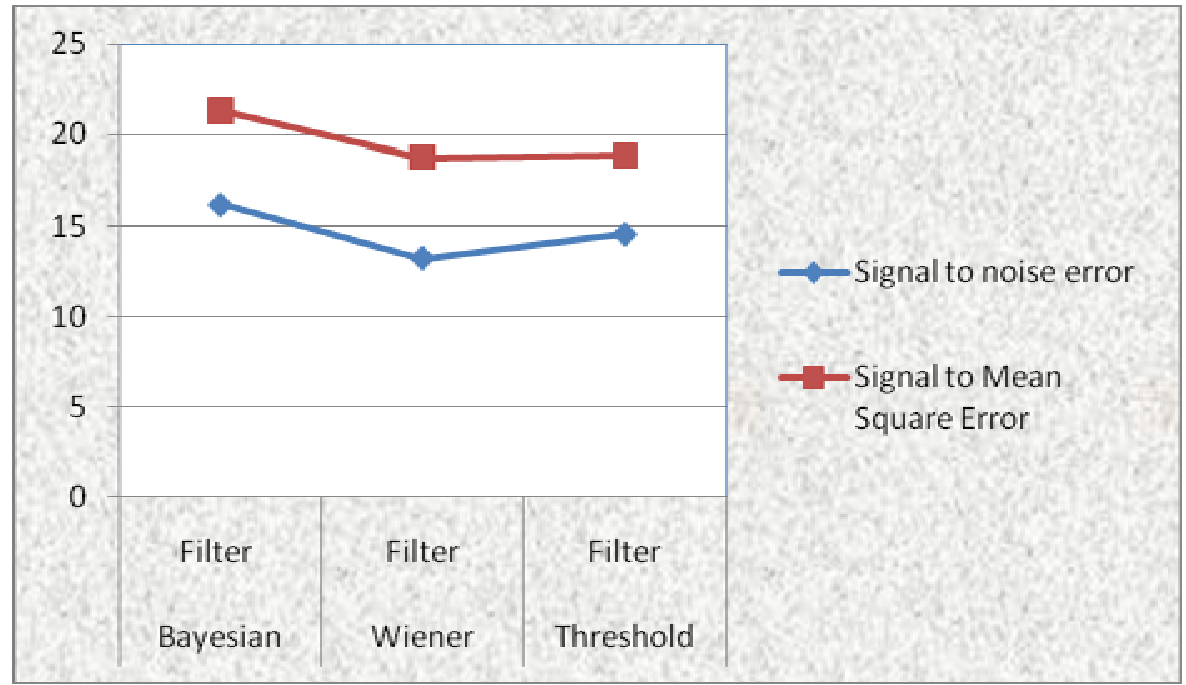

Chart 1. Comparision of various filters 


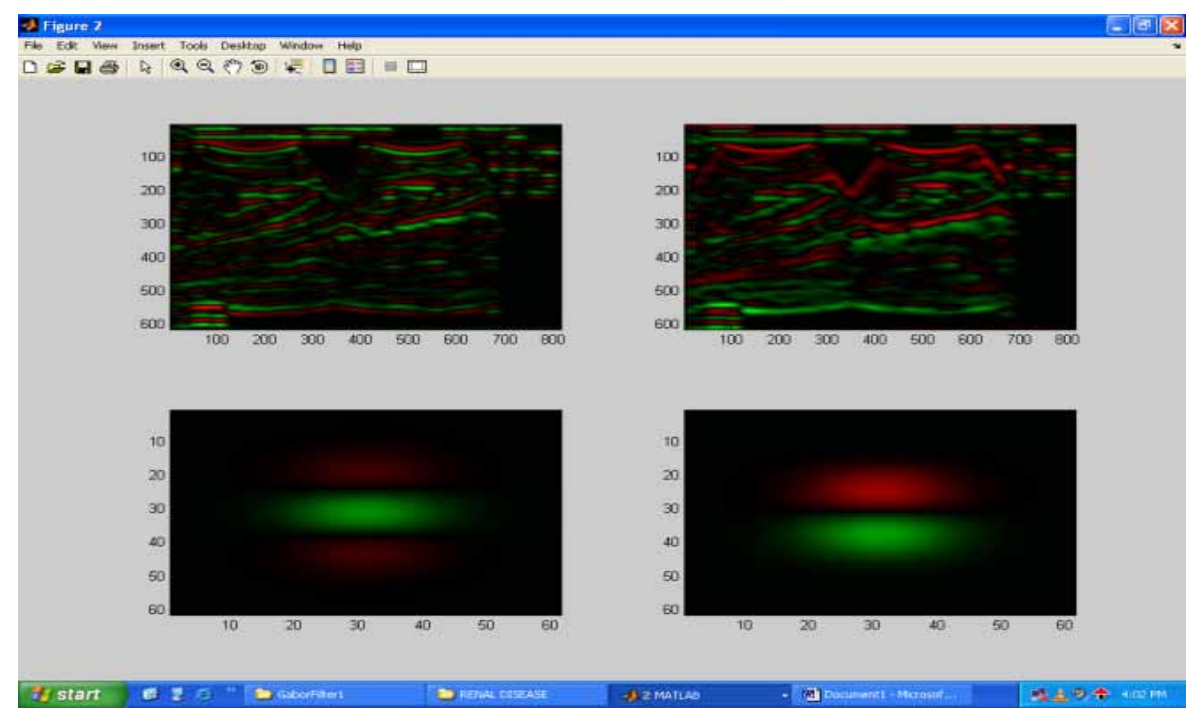

Figure 2. Texture variance of Gabor bank filter on US Kidney Image

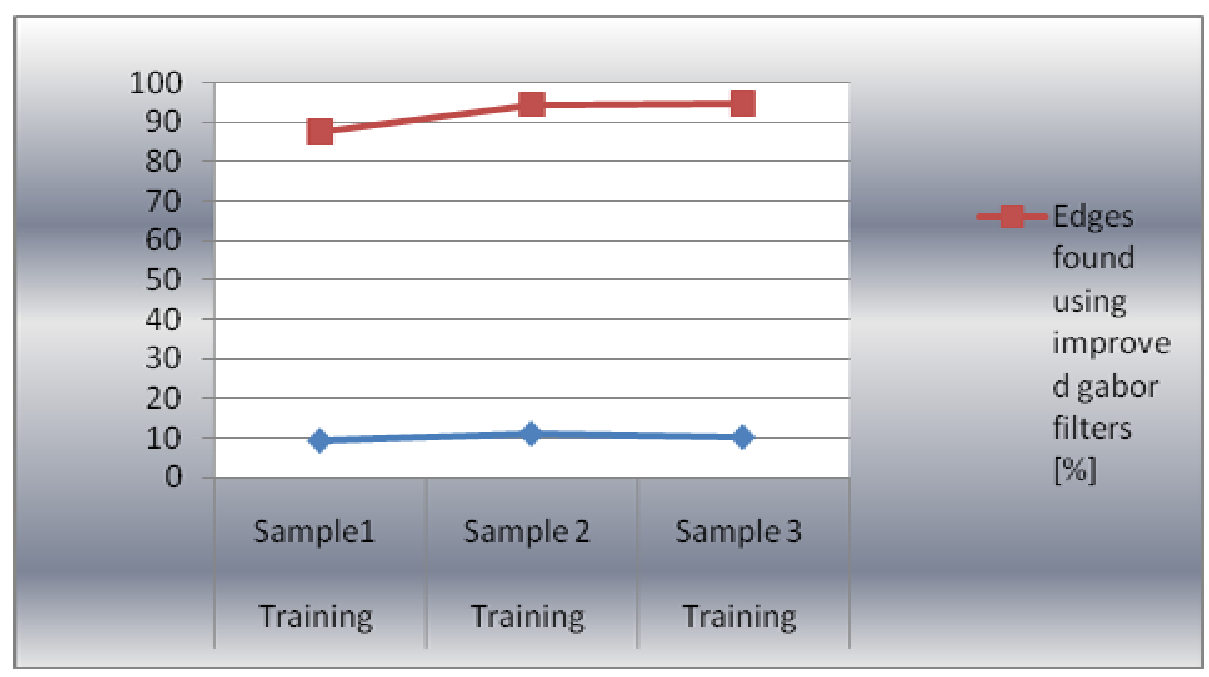

Chart 2. Edges found using improved gabor filters 